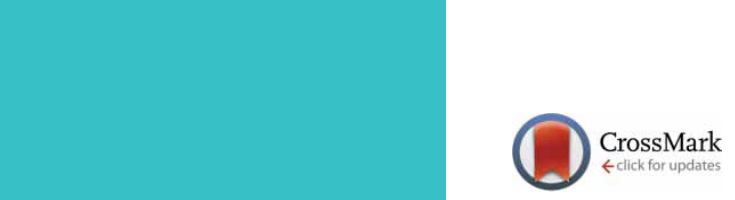

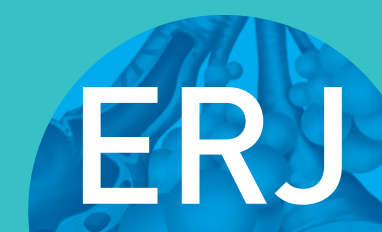

open research
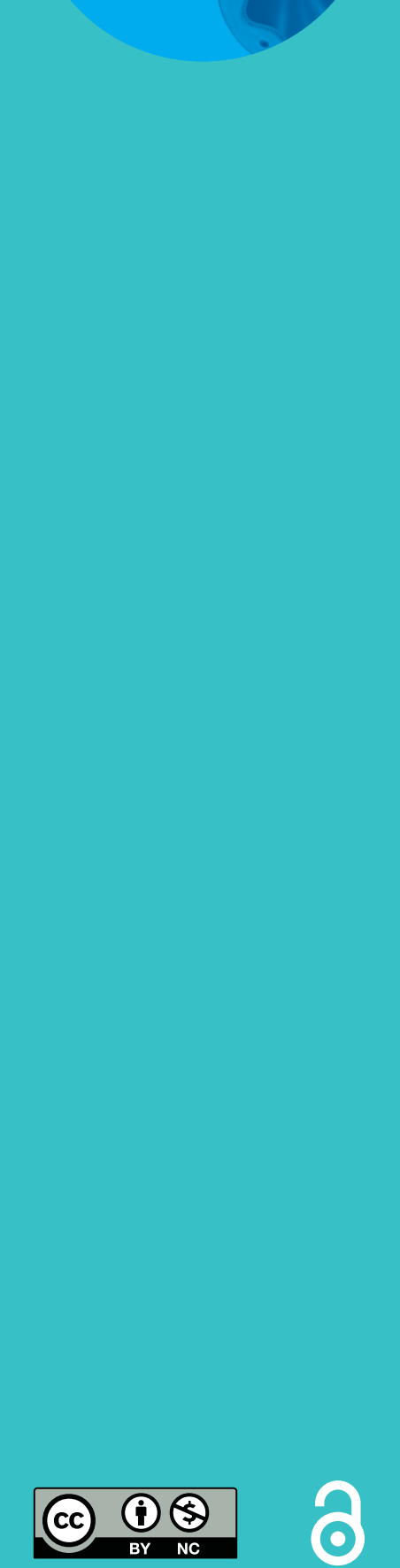

\section{ERS International Congress 2020: highlights from the Epidemiology and Environment Assembly}

\author{
Diana A. van der Plaat ${ }^{1}$, Aino K. Rantala ${ }^{2,3}$, Sheikh M. Alif $\mathbb{1}^{4}$, Dilek Karadoğan ${ }^{5}$, \\ Yutong $\mathrm{Cai}^{6}$ and Orianne Dumas $\mathbb{1}^{7}$
}

Affiliations: ${ }^{1}$ National Heart and Lung Institute, Imperial College London, London, UK. ${ }^{2}$ Center for Environmental and Respiratory Health Research, University of Oulu, Oulu, Finland. ${ }^{3}$ Dept of Chronic Diseases and Ageing, Norwegian Institute of Public Health, Oslo, Norway. ${ }^{4}$ Monash Centre for Occupational and Environmental Health, School of Public Health and Preventive Medicine, Monash University, Melbourne, Australia. ${ }^{5}$ Dept of Chest Diseases, School of Medicine, Recep Tayyip Erdoğan University, Rize, Turkey. ${ }^{6}$ Nuffield Dept of Women's and Reproductive Health, University of Oxford, Oxford, UK. ${ }^{7}$ Université ParisSaclay, UVSQ, Univ. Paris-Sud, Inserm, Équipe d’Épidémiologie respiratoire intégrative, CESP, 94807 Villejuif, France.

Correspondence: Orianne Dumas, Inserm, CESP, Équipe d'Épidémiologie respiratoire integrative, 16, avenue Paul Vaillant Couturier, 94807 Villejuif cedex, France. E-mail: orianne.dumas@inserm.fr

ABSTRACT In this article, early career members of the Epidemiology and Environment Assembly of the European Respiratory Society (ERS) summarise a selection of five sessions from the ERS 2020 Virtual International Congress. The topics covered include risk factors for chronic respiratory diseases over the life course, from early life origins to occupational exposures in adulthood, and the interplay between these risk factors, including gene-environment interactions. Novel results were also presented on smoking prevention and potential risks of vaping. Finally, the challenges and opportunities for epidemiological and environmental research brought by the coronavirus disease 2019 (COVID-19) pandemic were a major topic of this year's congress.

@ERSpublications

Highlights of \#ERSCongress 2020 from the Epidemiology and Environment Assembly https://bit. ly/3ogC1Me

Cite this article as: van der Plaat DA, Rantala AK, Alif SM, et al. ERS International Congress 2020: highlights from the Epidemiology and Environment Assembly. ERJ Open Res 2021; 7: 00849-2020 [https://doi.org/10.1183/23120541.00849-2020].

Received: 13 Nov 2020 | Accepted after revision: 13 Jan 2021

( ) The authors 2021. This version is distributed under the terms of the Creative Commons Attribution Non-Commercial Licence 4.0. For commercial reproduction rights and permissions contact permissions@ersnet.org 


\section{Introduction}

In this article, early career members of the Epidemiology and Environment Assembly of the European Respiratory Society (ERS) summarise a selection of results presented during the Society's 2020 virtual congress. Five sessions (four "oral presentation" sessions and one "hot topics" session) covering the main areas of interest of the Assembly, i.e. occupational, environmental and lifestyle determinants of respiratory health, and their interactions with genetic factors, are presented.

To address the complexity and multifactorial aetiology of chronic respiratory diseases such as asthma and COPD, a better understanding of their risk factors over the life course, from early life origins to occupational exposures in adulthood, and the interplay between these risk factors, including gene-environment interactions, is still needed. These important topics were largely covered during the congress, as highlighted in the current article. Novel results were also presented on smoking prevention and potential risks of vaping. Finally, the coronavirus disease 2019 (COVID-19) pandemic was a major topic of the congress. The pandemic brought new challenges and opportunities for epidemiological and environmental research, as illustrated in a session on air pollution and COVID-19.

\section{Origins of respiratory diseases: do early life exposure and diet really matter?}

Previous studies have suggested that respiratory diseases like asthma have origins in early life [1], but specific risk factors are not yet fully understood. This session highlighted some new and important findings on early risk factors of respiratory diseases and impaired lung function, covering environmental exposures like pollen and air pollution, maternal and child diet, fetal weight and bronchitis in early life.

First speaker Cecilia Lundholm hypothesised that the association between season of birth and risk of asthma and allergy [2] may be related to timing of exposure to pollen [3]. She and her colleagues studied the association of airborne allergenic pollen concentration (birch or alder plus hazel) in pregnancy and infancy with development of asthma during childhood in two Swedish register-based cohorts. Their findings suggested that exposure to high levels of pollen during infancy was associated with increased asthma incidence and current asthma at 6 years, while exposure during pregnancy might offer protection from childhood asthma. Seasonal timing and occurrence of infections might confound the association.

Previous studies have suggested that early life exposure to air pollution is associated with development of asthma up to adolescence [4], but there is limited information on the association with lung function growth. Two longitudinal cohort studies presented in this session investigated effects of air pollutant exposure to lung function development and both were conducted in European countries, where pollutant concentrations are relatively low [5, 6]. Qi Zhao reported findings from German GINIplus (German Infant Study on the influence of Nutrition Intervention PLUS environmental and genetic influences on allergy development) and LISA (Influence of Lifestyle-Related Factors on the Immune System and the Development of Allergies in Childhood) birth cohort studies, where he and his colleagues have investigated the effect of exposure to particulate matter with diameters $<2.5 \mu \mathrm{m}\left(\mathrm{PM}_{2.5}\right),<10 \mu \mathrm{m}\left(\mathrm{PM}_{10}\right)$ and nitrogen dioxide $\left(\mathrm{NO}_{2}\right)$ at the home addresses during first year of life on forced expiratory volume in $1 \mathrm{~s}\left(\mathrm{FEV}_{1}\right)$ and forced vital capacity (FVC) at ages 6, 10 and 15 years [5]. They found that early exposure to high levels of all the pollutants was associated with reduced lung function and the effect was significantly higher among children who were breastfed less than 12 months. Björn Lundberg presented a study on exposure to $\mathrm{PM}_{2.5}, \mathrm{PM}_{10}$ and $\mathrm{NO}_{2}$ based on individuals' residential addresses and lung function among infants living in Stockholm, Sweden [6]. They found a significant inverse relation between air pollution exposure and measured forced expiratory volume in $0.5 \mathrm{~s}$ and FVC at 6 months. Findings of these two studies suggest that exposure to air pollution in an area with comparatively low levels of air pollution may negatively affect lung function in infants and adolescents, leading to serious health consequences related to both obstructive and restrictive airway diseases later in life.

Two speakers presented findings on dietary exposures as risk factors of impaired lung function. Rosalie Mensink-Bout and her colleagues have performed an individual participant meta-analysis among 18326 mother-child pairs participating in the ALPHABET consortium (European consortium of seven longitudinal birth cohort studies) to investigate associations of maternal overall diet with child's respiratory health [7]. They used energy-adjusted Dietary Inflammatory Index (E-DII) score to estimate inflammatory potential and the Dietary Approaches to Stop Hypertension (DASH) score to estimate the overall quality of the maternal diet during pregnancy [8]. Results showed a modest association between suboptimal maternal diet and adverse respiratory health up to school age. Higher E-DII was associated with a lower FVC per interquartile range increase in the score and a very low DASH score $(<10$ th percentile) was associated with an increased risk of preschool wheezing and a very low $\mathrm{FEV}_{1} / \mathrm{FVC}$ ratio. The study presented by Mohammed Talaei provided new evidence on the longitudinal association between dietary intake of vitamin A and lung function [9]. He and his co-workers used the UK Avon Longitudinal Study of Parents and Children (ALSPAC) birth cohort data (3600 children) to investigate whether dietary intake 
of preformed vitamin A or its major provitamin, $\beta$-carotene, in mid-childhood is associated with lung function in adolescence. They found that higher intake of vitamin A at 7 years of age was associated with a higher $\mathrm{FEV}_{1}$ and forced expiratory flow at $25-75 \% \mathrm{FVC}\left(\mathrm{FEF}_{25-75}\right)$ at 15.5 years of age. No effect related to $\beta$-carotene was found. These findings indicate that the quality of maternal overall diet in pregnancy as well as dietary intake of vitamin A in childhood might play a role in child's respiratory outcomes.

Previous findings on the association between birth weight and risk of asthma are inconsistent [10]. Stephen Turner hypothesised that a reduced size during fetal life is more related to timing of asthma onset than asthma per se [11]. His study used routinely acquired information on hospital admissions and primary care prescribing data between 1987 and 2015 from almost 64000 children and their mothers. Reduced fetal size at first trimester was associated with earlier age at first hospital admission and reduced fetal size at third trimester and birth weight were both associated with earlier age at first asthma medication. Findings support their hypothesis although adverse health effects of preterm birth might also play a role in the association.

It is still unclear whether childhood bronchitis is associated with adverse lung health outcomes in middle-age adults [12]. Jennifer Perret presented a study that investigated the relationship between increasing severity of childhood bronchitis and lung health outcomes in the Tasmanian Longitudinal Health Study (TAHS), including altogether 3609 adults followed from birth to mean age of 53 years [13]. They defined parental-reported childhood bronchitis in severity categories as non-recurrent, recurrent non-protracted and recurrent protracted bronchitis by age of 7 years. They found significant dose-response trends between increased bronchitis severity and adult ever asthma and current asthma, ever pneumonia and worse adult lung function in an obstructed pattern. Findings suggest that childhood bronchitis may contribute to adult lung diseases especially if recurrent and protracted.

The findings presented in this session provide new knowledge on the role of early life exposures in the risk of chronic respiratory diseases using longitudinal approaches, which is important for understanding aetiology of complex diseases like asthma.

\section{Genetic and environmental influences on development of asthma and COPD}

In this session, a diverse range of studies were presented covering the genetic and environmental effects on lung health and lung disease. The presentations ranged from hardcore discovery genetics trying to identify SNPs associated with disease, to trying to unpick gene-environment interactions.

The first study presented by Catherine John identified and replicated eight genetic variants associated with co-existing asthma and COPD, including one variant that was not previously associated with asthma, COPD or lung function [14]. This is the largest genome-wide association study (GWAS) to date trying to investigate the genetics of asthma-COPD overlap (ACO) using data from 16 independent European and USA cohorts. Secondary analyses showed that the results were not driven by smoking and that ACO, asthma and COPD are genetically correlated $(r=0.74,0.83$ and 0.40 , respectively). These results thus provide evidence for a shared genetic environment but also implicate a possible distinct pathway for ACO development.

A second study presented by Stefania Ottaviani was able to identify 22 new and rare pathological mutations in the SERPINA1 gene, which can cause $\alpha_{1}$-antitrypsin deficiency (AATD) and subsequently can lead to lung emphysema and chronic liver disease [15]. Further characterisation showed that all, except one mutation, are located in the coding regions of the gene and that these mutations can cause protein deficiency or dysfunction (two null and 20 non-synonymous mutations). Interestingly, six patients had more than one deficient mutation and eight patients reported having a (lung) disease. Adding these new mutations to the diagnosis of AATD will aid the evaluation of the risk for lung disease.

Following these discovery genetic studies were two studies assessing the effect of environmental factors on gene expression. The first, a pharmacogenetic study presented by Elise Slob [16], identified multiple genetic variants affecting gene expression $(n=18)$ and DNA methylation levels $(n=57)$ in response to inhaled corticosteroids (ICS) use in airway wall biopsies from 42 COPD patients. Only one of these SNPs, associated with changes in DNA methylation and located in an intergenic region on chromosome 17, was also associated with an increased risk of exacerbations in asthmatic children treated with ICS. This indicates that ICS response is different between asthma and COPD and/or between adults and children. Nonetheless, the overlapping SNP could be further investigated to help predict ICS response in the future. The other study by Maria-Elisa Nordberg found that exposure to indoor particulate matter from nine moisture-damaged houses changed the expression of 43 genes in human airway epithelium cells [17]. However, the differential expression of these genes was not the same in every moisture-damaged house, which may indicate that the bioactive components of moist exposure may vary between houses. Interestingly, most of the identified genes were related to immunotoxicity and therefore the presenter 
would like to explore the interaction of the indoor microbiome of moisture damaged houses with the toxic responses in airway epithelium cells.

Finally, the last presenter attempted to assess the interaction between the environment and genetics using data from the large UK biobank. This study, presented by Diana van der Plaat, identified two rare genetic variants in the antioxidant genes GSTP1 and NOS1 that increased the risk of COPD by $19 \%$ and $30 \%$, respectively, when subjects were also exposed to occupational exposure such as vapours, gases, dusts and fumes (VGDF) [18]. Interestingly, this interaction effect was more apparent in never-smokers, whereas the effect of VGDF exposure without taking the interaction into account was only seen in smokers. This study showed that identifying gene-environment interactions is possible when using a targeted gene and case-only approach, but that larger studies are needed to perform genome-wide analyses.

Overall, this session showed that with large scale studies or specific disease characterisation, genetics can be used to unravel underlying mechanisms in lung pathology. Studying the combined effect of both the environment and genetics seems to be future of genetic epidemiologic research but it still has some considerable challenges to overcome both in design and scale [19]. However, it could help us to better understand complex diseases and the heterogeneity in disease risk.

\section{Workplace and outdoor air quality: from sensitisation to social media}

In this session, results were presented from long-term population-based and workplace-based cohorts, and case-control and cross-sectional studies, examining the impact of environmental and occupational exposures on respiratory health. The session covered several outcomes including cardiopulmonary mortality, occupational asthma, allergic diseases, lung function and idiopathic pulmonary fibrosis. In the field of occupational lung diseases, topics related to the exposure-response relationship and the use of social media tools were also discussed.

Two studies focussed on health effects of environmental pollutants. The first speaker Andrea Dalecká studied the effect of long-term air pollution on reduced lung function and cardiopulmonary mortality [20]. The German SALIA (Study on the Influence of Air Pollution on Lung, Inflammation and Aging) cohort data, including 2527 elderly women with a 22-year follow-up, were analysed. The lung function was measured with spirometry and reported using Global Lung Initiative (GLI) z-scores. Andrea Dalecka reported that exposures to nitrogen oxides $\left(\mathrm{NOx}\right.$ and $\mathrm{NO}_{2}$ ) were associated with an increased risk of cardiopulmonary mortality. The association between exposure to $\mathrm{PM}_{2.5}, \mathrm{PM}_{10}$ and cardiopulmonary mortality, was found to be mediated by lung function.

The second speaker, Isabella Annesi Maesano, presented a study investigating the association between fetal heavy metal exposure and the risk of asthma and allergic diseases in early childhood in the French EDEN birth cohort [21]. Higher levels of cadmium in cord blood were associated with an increased risk of childhood asthma and food allergy at 5 years of age, and higher levels of manganese were associated with eczema. No association between fetal exposure to lead in cord blood and allergic rhinitis was found.

Two studies were conducted in cohorts of workers. Rafael de la Hoz presented a study investigating the changes in body mass index (BMI) and expiratory flow in a cohort of 1329 former World Trade Center workers [22]. The participants had in total 6032 visits in the context of longitudinal surveillance, and at least two spirometries between 2002 and 2018. Mixed linear regression models were adjusted for sex, ethnicity, education, age at each visit, smoking status, arrival at the World Trade Center site within $48 \mathrm{~h}$, the baseline value for the predictor of interest (lung function and BMI) and the interval between test dates. De la Hoz reported bidirectional associations, suggesting that change in lung function and change in BMI were inversely associated with each other. Lidwien Smit presented a study on exposure-response relationships for platinum salt sensitisation in platinum refinery workers [23]. They used 17 years of retrospective data collected from 1614 workers, including personal air samples of six platinum refineries from South Africa, the UK and the USA. A decreasing trend in chloroplatinate exposure over the years was observed. A significant exposure-response relationship between cumulative and current chloroplatinates exposure and platinum salt sensitisation was found. Although the chloroplatinate exposure was decreasing over time, increasing trends of platinum salt sensitisation incidence were observed, indicating that a further reduction in exposure limit should be implemented.

Nicola Zivadinovic and colleagues investigated the association between occupational exposure and newonset asthma in Norway, using the 5-year follow-up data of the population-based Telemark study [24]. This analysis included 7120 participants aged 16-55 years in 2018, excluding asthmatics at baseline (2013). Zivadinovic reported a significant association between exposure to VGDF and new-onset asthma, where the exposures occurred weekly or more frequently in the past 12 months. The risk of new-onset asthma was also increased with more frequent VGDF exposure, suggesting a dose-response trend. The study also 
found a population attributable fraction of $17 \%$, which is consistent with recent estimates of the occupational burden of asthma [25].

Occupational exposure to asbestos is associated with idiopathic pulmonary fibrosis (IPF), as found in previous studies [26, 27]. Carl Reynolds investigated the relationship between occupational exposure to asbestos, cigarette smoking, MUC5B rs35705950 genotype and the risk of IPF [28]. This UK multicentre case-control study included 494 cases and 466 controls from 21 hospitals. Asbestos exposure was assessed using a job exposure matrix. Neither smoking nor occupational exposure to asbestos was individually associated with IPF. However, asbestos exposure interacted with smoking and the minor allele of the $M U C 5 B$ promoter variant to increase IPF risk. This potential gene-environment interaction emphasises the importance of host susceptibility in IPF.

Finally, David Fishwick talked about the use of digital and social media to highlight the risks of occupational exposures as a cause of lung diseases [29]. The ERS, in collaboration with the European Lung Foundation, has developed an online tool to increase lung health awareness. The participants were invited through a Facebook advertising campaign targeting at-risk workers to participate in an online quiz. A total of 22393 individuals participated. The commonly reported symptoms were nasal symptoms (47\%), cough (42\%), chest tightness $(21 \%)$ and wheeze $(20 \%)$. For $37.6 \%$ of the participants, at least one of the respiratory symptoms were work-related, and $6.1 \%$ had changed their job due to breathing issues. Jobs with high levels of reported wheeze included work in a sports centre, construction site, factory work and workplaces where a high chemical exposure may have occurred. Wheeze was also associated with exposure to animals, aerosols, dyes, asbestos, cleaning agents, latex, chlorine and fumes. This tool has provided an insight into workplace exposure experienced by the workers and proved to be an effective resource to understand exposures in high-risk industries.

Overall, the findings presented in this session highlight the importance of investigating environmental and workplace exposures using long-term follow-up cohorts, which is beneficial for example to understand trends over time.

\section{From tobacco and vaping health effects to tobacco cessation}

In this section, recent results regarding the health effects of vaping and the impact of smoking cessation were presented.

Three studies focussed on health impacts of electronic cigarette (e-cigarette). The first study, presented by Hanno Erythropel, looked at differences in flavourant levels and synthetic coolant use between USA, European Union (EU), and Canadian Juul e-cigarettes [30]. An increase in sale of Juul between 2014 and 2019 in US markets has been reported [31]. In the EU, nicotine content of any e-liquid was limited to $1.7 \%$, but was found to be higher in USA and Canada markets [32]. Erythropel and his colleagues evaluated and compared the flavour contents (mint, vanilla and mango) of e-liquids. Quantification of principal flavorants per country showed that, in the EU products, principal flavours were reduced. However, mint (menthol) component in EU products was compensated by the synthetic coolant WS3. Another study, presented by Sven-Eric Jordt, examined the effect of flavour-solvent reaction products in e-cigarette liquids on airway epithelial cell [33]. E-cigarette liquids consist of different flavour chemicals and aldehydes. In a previous study from this group, it was discovered that aldehydes can react with propylene glycol, a major solvent in e-cigarette, to form acetal compounds [34]. In the current study, the toxicity of these newly found compounds on cultured bronchoepitelial cells was evaluated using live/dead assays, and compared with benzaldehyde. They found that propylene glycol acetal was more cytotoxic than benzaldehyde alone. In metabolic assay these aldehyde acetals were found to suppress ATP production of mitocondria more strongly than the parent aldehydes. The authors concluded that the toxicity of these compounds emphasise the need for new risk assessment. Karin Laursen then presented a randomised controlled exposure study on passive exposure to vape among COPD patients [35]. Because of the increasing use of e-cigarettes worldwide, passive vape exposure is common, even in smoke-free areas [36]. The study aimed to investigate local and systemic effects of short-term passive exposure among patients with mild or moderate COPD. A randomised controlled cross-over exposure study was conducted in 16 patients. All patients were exposed to two sessions, clean air and passive vape from e-cigarette, lasting $4 \mathrm{~h}$ each. Surfactan protein A in exhaled air was found to be negatively affected by exposure to vape. Several plasma proteins increased significantly after vape exposure compared to clean air exposure.

Although studies have found that smoking cessation or reduction is associated with a lower risk of lung cancer, compared with heavy smoking, studies on the link between low intensity smoking and respiratory diseases and lung cancer mortality are lacking. Pallavi Balte presented a study aimed to address this question in a large US population-based cohort of 18730 participants with median follow up of 17 years [37]. Compared with nonsmokers, light smokers (fewer than 10 cigarettes per day) had 2.5-times increased risk 
of respiratory diseases mortality and about nine-times increased risk of lung cancer mortality. Rachel Gemine presented a cohort study on treatment complication rates in continued smokers versus quitters after a diagnosis of lung cancer [38]. In a previous study, GEmine et al. [39] found that a year after lung cancer diagnosis, $69 \%$ of quitters were alive, compared with $53 \%$ of continued smokers $(\mathrm{p}<0.01)$. The current study included 1134 patients with newly diagnosed non-small cell lung cancer in a UK multicentre study. Among all patients recruited, 290 (25.6\%) were smokers at baseline and 84 (29\%) of them quit within the first 3 months following the diagnosis. One month after the diagnosis, quitters had fewer treatment complications than those who continued to smoke $(p=0.03)$; however, this was not seen at 6 th or 12 th months.

Lutz Siemer presented preliminary results of a RCT on smoking cessation comparing blended with face-to-face treatment delivery mode [40]. The setting was an outpatient smoking cessation clinic in a Dutch hospital and the sample size was 344 patients. High-intensity smoking cessation treatment was performed for 6 months, including cognitive behavioural counselling, and if necessary, pharmacotherapy and nicotine replacement therapy. Face-to-face treatment included 10 face-to-face sessions, while blended treatment consisted of five face-to-face and five web-based sessions. Intention-to-treat analysis was performed. Cotinine-validated quit rates at 3 months were lower in the blended treatment arm compared with face-to-face ( $4.8 \%$ versus $17.5 \%)$, but was not significantly different at $5 / 6$ months. Overall, these prelimiary results were suggestive of an inferiority of the blended treatment.

The last study, presented by Linnea Hedman, aimed to look at the association between age at asthma onset and initiation of daily smoking [41]. In this prospective cohort study, children were recruited at age 8 years, and followed until 28 years of age. By age 28 years, 18\% ( $n=603)$ had reported ever having physician-diagnosis of asthma in any of the questionnaires. Among them, $49 \%$ had onset at age 12 years or younger (early onset). Among asthmatics, smoking rate was higher (23.6\%) than among those never reporting asthma (19.4\%). The association between early onset asthma and smoking remained significant after adjustment for potential confounders. The authors concluded that having early onset asthma did not counteract smoking initiation.

In conclusion, the presented studies revealed differences in e-cigarette compounds among international markets and demonstrated the health hazards of e-cigarette liquids both at experimental and at clinical levels. Exposure to cigarette smoke has been associated with respiratory diseases' worse outcomes. By also evaluating the effectiveness of novel tobacco cessation methods, the session has covered the area with a wide perspective.

\section{What we know and what we need to know about air pollution and COVID-19}

COVID-19, caused by severe acute respiratory syndrome coronavirus 2 (SARS-CoV-2), was declared a pandemic on 11th March 2020 by World Health Organization. While the pandemic is still unfolding, research has emerged to investigate the potential interaction between air pollutants and SARS-CoV-2, as well as the relationship between short- or long-term air pollution exposures and COVID-19 risk. This symposium was to present the latest scientific evidence up to the time of the ERS International Congress in early September 2020, highlighting key research needs that may improve our understanding of this rapidly evolving research.

The first speaker, Sara De Matteis, presented the latest evidence on an important research question: is COVID-19 mortality associated with long-term air pollution exposure? [42]. OgEN et al. [43], who analysed the relationship between COVID-19 case fatality and nitrogen dioxide $\left(\mathrm{NO}_{2}\right)$ during January and February 2020 across 66 regions in Italy, Spain, Germany and France. This ecological study concluded that almost $80 \%$ of fatality cases were from highly polluted regions, including northern Italy and Madrid. In a pre-print paper by $\mathrm{WU}_{\mathrm{U}}$ et al. [44], it was found that every $1 \mathrm{ug} \cdot \mathrm{m}^{-3}$ increase in fine particulate matter $\left(\mathrm{PM}_{2.5}\right)$ (averaged between 2000-2016) was associated with 15\% (95\%CI: 5\%-25\%) increase in the COVID-19 death rate up to 22 April, 2020, across more than 3000 counties in the USA, adjusting for 17 potential confounding factors. Interestingly, this estimation was later updated, in which additional three potential confounding factors including timing of the epidemic's spread, timing of the social distancing policies and the population age distribution were adjusted, the risk was downscaled to 8\% (95\% CI 2\%-15\%). Liang et al. [45] averaged long-term exposure to $\mathrm{PM}_{2.5}, \mathrm{NO}_{2}$ and ozone from 2010-2016 and analysed their relationships with COVID-19 case-fatality and mortality rates from January to July, 2020, in more than 3000 counties in the USA. Whilst no associations with either $\mathrm{PM}_{2.5}$ or ozone in the multi-pollutants models were found, they reported that every interquartile range increase in $\mathrm{NO}_{2}(4.6 \mathrm{ppb})$ was associated with $11.3 \%$ (95\% CI 4.9\%-18.2\%) and 16.2\% (95\% CI 8.7\%-24\%) increase in case-fatality and mortality rate respectively, in co-pollutant models. In another ecological study also at county-level in the USA, higher exposure to diesel particulate matter (averaged 2014-2019) was significantly associated with COVID-19 prevalence and fatality rates [46]. The study also suggested that air pollution from novel 
sources such as waste treatment and disposal facilities may have a role in impacting COVID-19 outcomes. These studies were all of ecological design, therefore ecological fallacy was unavoidable. There are still unsolved issues in terms of defining COVID-19 mortality; for example, no exposed population was known or no standardisation on age or sex was used. Limited or no adjustments were made on the individual-level confounding factors, nor the spatial-temporal correlation. Last but not least, other potential confounding factors such as population density and mobility, social interaction and basic reproduction number should be considered carefully.

Francesco Forastiere then addressed the research needs on air pollution and COVID-19 [47]. In addition to the limitations noted by Sara de Matteis, he described more caveats to interpret findings from ecological studies. First, testing rates are also highly variable across countries and within countries. Second, calculating and reporting practices for cases and deaths are highly variable across countries. Third, a potential confounding by indication should be considered, as lockdown measures due to high COVID-19 infection rate may have improved air quality. Fourth, spatial correlation (e.g. person-to-person transmission) is often difficult to adjust for. All current ecological studies are lacking adequate consideration of a range of community, environmental and individual factors relating to both the endemic component (i.e. the spark of infection in the community) and the epidemic component (i.e. how infection spreads in the community and beyond). Research opportunities to address the current knowledge gap include cohort studies of general population to assess the cumulative incidence of infection, case-fatality and mortality, or cohort studies of patients to evaluate disease severity. Also, a well-designed case-control study may provide early, valuable insights [48]. Future research should address three important methodological aspects: spatial correlation at both community and individual-level; an extensive search of factors that might explain the epidemic pattern but may also be linked to air pollution; and probability of getting a COVID-19 test when incidence is the study outcome [49].

Finally, Barbara Hoffmann gave an interesting talk on whether the COVID-19 lockdown could be used to conduct air pollution accountability research [50]. An accountability research on air pollution typically compares air quality and population health before and after an introduction of a policy or an unintentional change in air quality [51]. Accountability research is valuable in many aspects, including but not limited to, documenting the success of regulatory actions, assessing cost-benefit of such actions, and validating burden of disease estimations in "real-world' scenarios. One example is the implementation of Dublin Coal Ban in 1990 [52]. While accountability research is a valuable instrument, it has some important methodological challenges in the case of COVID-19 lockdown. First, air pollution emissions were generally reduced in different extent due to decreased economic activities when lockdown measures were in place. However, some specific emissions such as household emissions may have increased during the same period. Globally, declines of ground-level air pollution have been recorded in many countries [53], but such declines were not necessarily uniform at local level within the countries. These highlight the difficulties in assessing the exposure concentration at individual level. Second, in addition to air quality changes, lockdown measures have also brought in drastic changes to individual socioeconomic positions and lifestyle factors, implications from which on health could potentially be substantial and thus should be comprehensively assessed. Also, assessment of health outcomes might even have changed during lockdown; for example, fewer cases of certain categories of chronic diseases may be registered as people were reluctant or discouraged to visit hospital during the pandemic. The speaker therefore acknowledged that it will be challenging to execute the air pollution accountability research using COVID-19 lockdown.

Two additional points were made by Forastiere to close this symposium. First, the scientific community should conduct the research on this topic with care and responsibility [54]. The research should be underpinned by strong research hypotheses, robust study design and statistical approaches, acknowledged limitations and a rigorous peer-review process. Second, the pandemic presents an opportunity to cities worldwide on how to reform the urban transport system to discourage car use and increase public spaces [55], which are beneficial to both the environment and human health in the long term.

In summary, currently available epidemiological evidence deems to be of low quality, indicating more rigorous studies preferably at individual-level are needed to understand whether air pollution enhances susceptibility to infection, and/or increases severity of COVID-19 outcomes.

\section{Conclusion}

High-quality research in the field of respiratory and environmental epidemiology was presented during the ERS Virtual International Congress, and the present article highlighted a selection from a large amount of remarkable sessions. Novel findings emphasise the role of early life environment in the aetiology of chronic respiratory diseases, including modifiable risk factors, such as overall maternal diet. Large studies revealed new genetic variants for asthma-COPD overlap, and gene-environment interactions in COPD for common occupational exposures (vapours, dusts, gases and fumes). Promising results were presented 
regarding the use of social media and targeted online tools both to collect data and raise awareness on occupational lung diseases. Research challenges and opportunities related to the COVID-19 pandemics, discussed in the context of air pollution studies, are relevant for many other environmental and lifestyle risk factors of interest for the Epidemiology and Environment Assembly of the ERS.

Conflict of interest: D.A. van der Plaat has nothing to disclose. A.K. Rantala has nothing to disclose. S.M. Alif has nothing to disclose. D. Karadoğan has nothing to disclose. Y. Cai has nothing to disclose. O. Dumas has nothing to disclose.

Support statement: D.A. van der Plaat has received funding from the European Respiratory Society and the European Union's H2020 Research and Innovation Programme under Marie Sklodowska-Curie grant agreement number 713406. A.K. Rantala has received funding from the European Respiratory Society (Long-Term Research Fellowship 2019). Funding information for this article has been deposited with the Crossref Funder Registry.

\section{References}

1 Duijts L. Fetal and infant origins of asthma. Eur J Epidemiol 2012; 27: 5-14

2 Almqvist C, Ekberg S, Rhedin S, et al. Season of birth, childhood asthma and allergy in a nationwide cohortMediation through lower respiratory infections. Clin Exp Allergy 2020; 50: 222-230

3 Lundholm C, Almqvist C, Ekberg S, et al. Pollen exposure in pregnancy and infancy and risk of childhood asthma. Eur Respir J 2020; 55: Suppl. 65, 4981.

4 Gehring U, Wijga AH, Hoek G, et al. Exposure to air pollution and development of asthma and rhinoconjunctivitis throughout childhood and adolescence: A population-based birth cohort study. Lancet Respir Med 2015; 3: 933-942.

5 Zhao Q, Markevyc I, Berdel D, et al. Early exposure to air pollution and lung function development into adolescence: The GINIplus/LISA birth cohorts. Eur Respir J 2020; 55: Suppl. 65, 4982.

6 Lundberg B, Gruzieva O, Eneroth K, et al. Impaired infant lung function in relation to air pollution exposure. Eur Respir J 2020; 55: Suppl. 65, 4983.

7 Mensink-Bout R, van Meel E, de Jongste JC, et al. Maternal diet in pregnancy and child's respiratory outcomes: an individual participant data meta-analysis. Eur Respir J 2020; 55: Suppl. 65, 4984.

8 Aubert AM, Forhan A, de Lauzon-Guillain B, et al. Deriving the dietary approaches to stop hypertension (DASH) score in women from seven pregnancy cohorts from the European alphabet consortium. Nutrients 2019; 11: 2706

9 Talaei M, Lietz G, Granell R, et al. Dietary vitamin A intake and lung function in childhood: longitudinal study. Eur Respir J 2020; 55: Suppl. 65, 4985.

10 Källén B, Finnstrom O, Nygren KG, et al. Association between preterm birth and intrauterine growth retardation and child asthma. Eur Respir J 2013; 41: 671-676.

11 Turner S, Aucott L. Reduced antenatal and birth size and time to onset of asthma. Eur Respir J 2020; 56: 4986.

12 Bui DS, Walters HE, Burgess JA, et al. Childhood respiratory risk factor profiles and middle-age lung function: A prospective cohort study from the first to sixth decade. Ann Am Thorac Soc 2018; 15: 1057-1066.

13 Perret JL, Lowe AJ, Wurzel D, et al. Childhood bronchitis and adult respiratory outcomes: a prospective study from age 7 to 53 years. Eur Respir J 2020; 55: Suppl. 65, 4987.

14 John C, Guyatt AL, Olafsdottir T, et al. A genome-wide association study of asthma-COPD overlap syndrome (ACOS). Eur Respir J 2020; 55: Suppl. 65, 4989.

15 Ottaviani S, Fra AM, Balderacchi AM, et al. Identification and characterisation of twenty-two novel SERPINA1 pathological mutations. Eur Respir J 2020; 55: Suppl. 65, 4922.

16 Slob EMA, Faiz A, Vijverberg S, et al. Bronchial airway inducible expression and methylation QTL mapping identifies a single nucleotide polymorphism predicting inhaled corticosteroids response heterogeneity. Eur Respir J 2020; 55: Suppl. 65, 4920.

17 Nordberg M-E, Täubel M, Leppänen $\mathrm{H}$, et al. Activation of toxicology-related genes in human airway constructs after exposure to indoor air particulate matter from moisture-damaged houses. Eur Respir J 2020; 55: Suppl. 65, 4923.

18 van der Plaat DA, De Matteis S, Sadhra S, et al. Interaction between VGDF exposure and antioxidant genes on COPD in UK Biobank. Eur Respir J 2020; 55: Suppl. 65, 4921.

19 Duggal P, Ladd-Acosta C, Ray D, et al. The evolving field of genetic epidemiology: from familial aggregation to genomic sequencing. Am J Epidemiol 2019; 188: 2069-2077.

20 Dalecká A, Wigmann $\mathrm{C}$, Altrug $\mathrm{H}$, et al. The mediating role of lung function on air pollution-induced cardiopulmonary mortality. Eur Respir J 2020; 55: Suppl. 65, 3846.

21 Pesce G, Baiz N, Huel G, et al. Foetal exposure to heavy metals and risk of asthma and allergic diseases in early childhood: a population-based birth-cohort study. Eur Respir J 2020; 55: Suppl. 65, 3847.

22 Alruwaily A, Sood A, de la Hoz R, et al. Change in body mass index and expiratory flow in World Trade Center workers. Eur Respir J 2020; 55: Suppl. 65, 3848

23 Smit LA, Jacobs J, Portengen L, et al. Exposure-response relationships for platinum salt sensitization in platinum refinery workers: a 17-year retrospective study. Eur Respir J 2020; 55: Suppl. 65, 3849.

24 Zivadinovic N, Klepaker G, Svendsen M V, et al. Occupational exposure and new onset asthma in the Telemark study: a five-year follow up. Eur Respir J 2020; 55: Suppl. 65, 3850.

25 Blanc PD, Annesi-maesano I, Balmes JR, et al. The occupational burden of nonmalignant respiratory diseases. An official American Thoracic Society and European Respiratory Society statement. Am J Respir Crit Care Med 2019; 199: $1312-1334$

26 Walters GI. Occupational exposures and idiopathic pulmonary fibrosis. Curr Opin Allergy Clin Immunol 2020; 20: 103-111.

27 Abramson MJ, Murambadoro T, Alif SM, et al. Occupational and environmental risk factors for idiopathic pulmonary fibrosis in Australia: Case-control study. Thorax 2020; 75: 864-869. 

smoke, and MUC5B promoter polymorphism rs35705950. Findings from the idiopathic pulmonary fibrosis job exposures study (IPFJES). Eur Respir J 2020; 55: Suppl. 65, 3851.

29 Fishwick D, Thompson E, Heederik D, et al. Using digital and social media to highlight the risks of occupational exposures as a cause of lung diseases. Eur Respir J 2020; 55: Suppl. 65, 3852.

30 Erythropel HC, Anastas PT, Krishnan-Sarin S, et al. Late Breaking Abstract - Differences in flavorant levels and synthetic coolant use between USA, EU and Canadian Juul products. Eur Respir J 2020; 55: Suppl. 65, 4382.

31 Juul and Youth: Rising E-Cigarette Popularity [cited]. www.tobaccofreekids.org/assets/factsheets/0394.pdf Date last accessed: October 28, 2020; date last updated: February 4th, 2021.

32 Erythropel HC, Anastas PT, Krishnan- S, et al. Differences in flavourant levels and synthetic coolant use between USA, EU and Canadian Juul products. Tob Control 2020. Epub ahead of print: doi:10.1136/ tobaccocontrol-2

33 Jordt SE, Caceres AI, Erythropel HC, et al. Flavor-solvent reaction products in electronic cigarette liquids activate respiratory irritant receptors and elicit cytotoxic metabolic responses in airway epithelial cell. Eur Respir J 2020; 55: Suppl. 65, 4384.

34 Erythropel HC, Jabba S V, Dewinter TM, et al. Original investigation formation of flavorant: propylene glycol adducts with novel toxicological properties in chemically unstable e-cigarette liquids. Nicotin Tob Res 2020; 21 : 1248-1258.

35 Laursen KR, Bønløkke JH, Bilde M, et al. Acute health effects after passive e-vape among patients with COPD an RCT exposure study. Eur Respir J 2020; 55: Suppl. 65, 4387

36 Hammond D, Reid JL, Rynard VL, et al. Prevalence of vaping and smoking among adolescents in Canada, England, and the United States : repeat national cross sectional surveys. BMJ 2019; 10: 12219.

37 Balte $\mathrm{P}$, Bhatt S, Chaves P, et al. Association of low-intensity smoking with respiratory and lung cancer mortality. Eur Respir J 2020; 55: Suppl. 65, 4389.

38 Lanyon K, Gemine RE, Davies G, et al. Treatment complication rates in continued smokers vs quitters after a diagnosis of lung cancer: a cohort study. Eur Respir J 2020; 55: Suppl. 65, 4383.

39 Gemine RE, Ghosal R, Collier G, et al. Longitudinal study to assess impact of smoking at diagnosis and quitting on 1-year survival for people with nonsmall cell lung cancer. Lung Cancer 2019; 129: 1-7.

40 Siemer L, Pieterse ME, Ben Allouch S, et al. Outpatient smoking cessation: preliminary findings of a non-inferiority RCT comparing blended with face-to-face delivery mode. Eur Respir J 2020; 55: Suppl. 65 , 4385.

41 Hedman L, Backman H, Andersson M, et al. Asthma do not counteract smoking initiation. Eur Respir J 2020; 55 Suppl. 65, 4386.

42 De Matteis S. Is COVID-19 mortality related to long-term air pollution exposure? Eur Respir J 2020; 55: Suppl. 65, 5265.

43 Ogen Y. Assessing nitrogen dioxide (NO2) levels as a contributing factor to coronavirus (COVID-19) fatality Sci Total Environ 2020; 726: 138605.

44 Wu X, Nethery RC, Sabath BM, et al. Air pollution and COVID-19 mortality in the United States: Strengths and limitations of an ecological regression analysis. Sci Advance 2020; 6: eabd4049.

45 Liang D, Shi L, Zhao J, et al. Urban Air Pollution May Enhance COVID-19 Case-Fatality and Mortality Rates in the United States. Innovation (N Y) 2020; 1: 100047.

46 Hendryx M, Luo J. COVID-19 prevalence and fatality rates in association with air pollution emission concentrations and emission sources. Environ Pollut 2020; 265: 115126.

47 Forastiere F. Research needs and opportunities on air pollution and COVID-19. Eur Respir J 2020; 55: Suppl. 65, 5268 .

48 Vandenbroucke JP, Brickley EB, Vandenbroucke-Grauls CMJE, et al. A Test-Negative Design with Additional Population Controls Can Be Used to Rapidly Study Causes of the SARS-CoV-2 Epidemic. Epidemiology 2020; 31 : $836-843$.

49 Nguyen LH, Drew DA, Graham MS, et al. Risk of COVID-19 among front-line health-care workers and the general community: a prospective cohort study. Lancet Public Heal 2020; 5: e475-e483.

50 Hoffmann B. Can the Corona lockdown be used for accountability research? Chances, challenges and caveats. Eur Respir J 2020; 55: Suppl. 65, 5267

51 Rich DQ. Accountability studies of air pollution and health effects: lessons learned and recommendations for future natural experiment opportunities. Environ Int 2017; 100: 62-78

52 Clancy L, Goodman P, Sinclair H, et al. Effect of air-pollution control on death rates in Dublin, Ireland: an intervention study. Lancet 2002; 360: 1210-1214.

53 Venter ZS, Aunan K, Chowdhury S, et al. COVID-19 lockdowns cause global air pollution declines. Proc Natl Acad Sci U S A 2020; 117: 18984-18990.

54 Heederik DJJ, Smit LAM, Vermeulen RCH. Go slow to go fast: a plea for sustained scientific rigour in air pollution research during the COVID-19 pandemic. Eur Respir J 2020; in press [doi: 10.1183/13993003.013612020].

55 Acuto M. COVID-19: Lessons for an Urban(izing) World. One Earth 2020; 2: 317-319. 